\title{
Metastatic adrenal carcinoma masquerading as primary bronchial carcinoma: report of two cases
}

\author{
ALISON CE MCCARTNEY \\ From the Department of Histopathology and Experimental Pathology, Charing Cross Hospital, London
}

\begin{abstract}
A single, intrabronchial metastasis of adrenal cortical carcinoma is a rare presentation of a rare tumour. Two cases are reported where the lesions clinically and radiologically appeared to be primary bronchial carcinomas. Initial histological diagnosis was difficult but the two tumours had similar, distinctive histological appearances.
\end{abstract}

\section{Case reports}

CASE 1

A 25 year old woman presented with a three month history of dyspnoea and weight loss. Eight months previously she had had a single episode of haemoptysis, associated with a productive cough with mucoid sputum; this had been treated with antibiotics. She was a non-smoker. Further questioning revealed an eight month history of acne, hirsutism, and apparent infertility.

On examination she was tachypnoeic, with reduced air entry in the left upper zone, dullness to percussion, and diminished movements of the left chest wall. She had facial hair which required daily shaving and a "male" pubic escutcheon. A chest radiograph showed elevation of the left hemidiaphragm, pleural effusion, partial occlusion of the left main bronchus, and collapse of the left lower lobe. The appearances strongly suggested primary carcinoma of the lung. Tomography showed intrabronchial obstruction of the left main bronchus $3 \mathrm{~cm}$ from its origin and underlying consolidation. At fibreoptic bronchoscopy a soft creamy tumour was seen. Biopsy showed extensive necrosis; some clumps of malignant cells were present but not sufficient for a firm diagnosis. Rigid bronchoscopy and subsequently thoracotomy were performed. The tumour enveloped the left pulmonary artery and aorta and had infiltrated the pericardium. The tumour was composed of plump, polygonal, eosinophilic cells with appreciable nuclear pleomorphism, arranged around delicate sinusoidal blood vessels (fig 1). Electron microscopy showed lipochrome pigment, tubular mitochondria, and intracytoplasmic vacuolation (fig 2). A diagnosis of metastatic adrenal carcinoma was made. The urine dehydroepiandrosterone (DHA) screening test was positive and 24 hour urine analysis showed the DHA concentration to be $80 \mu \mathrm{mol} / 24 \mathrm{~h}$ (normal $0.7-1.75 \mu \mathrm{mol} / 24 \mathrm{~h}$ ), the $17 \mathrm{oxy}$ steroid concentration $1240 \mu \mathrm{mol} / 24 \mathrm{~h}$ (normal 15-

Address for reprint requests: Department of Histopathology and Experimental Pathology, Charing Cross Hospital, London W6 8RF.

Accepted 2 November 1983
$70 \mu \mathrm{mol} / 24 \mathrm{~h})$, and the free cortisol concentration $3660 \mathrm{nmol} / 24 \mathrm{~h}$ (normal $<275 \mathrm{nmol} / 24 \mathrm{~h}$ ). The plasma testosterone concentration was $9.7 \mathrm{nmol} / \mathrm{l}$ (normal $0.5-$ $2.5 \mathrm{nmol} / \mathrm{l}$ ). Computed tomography and intravenous pyelography showed a mass $8 \times 4 \times 4 \mathrm{~cm}$ surrounding the inferior vena cava. She was treated with $o^{\prime} p^{\prime}$ DDD (1, 1-dichloro-2-(o-chlorophenyl)-2-( $p$-chlorophenyl) ethane), cisplatin (cis-diammine dichloroplatinum), and trilostane (Modrenal). This treatment led to diminution of the testosterone and cortisol concentration but no regression of the primary tumour mass was seen at repeat scanning. Necropsy 13 months later showed multiple hepatic and pulmonary metastases from a large left sided, partly necrotic adrenal cortical carcinoma. The right adrenal and both ovaries were atrophic, with no evidence of metastases.

\section{CASE 2}

A 55 year old man was seen at the University Hospital of Siena. Chest radiography showed a left upper lobe lesion. Bronchoscopy gave a negative result but a bronchogram suggested a more distal obstruction. A tumour was resected at thoracotomy; a firm histological diagnosis was not made but the lesion was thought to be malignant. Ten months later the patient underwent laparotomy for renal colic and biopsy specimens were taken of a retroperitoneal suprarenal mass $12 \times 5 \times 2 \mathrm{~cm}$ (this mass had not been palpable at the time of the initial operation). Histological sections of both lesions were sent to this hospital.

The sections from the lung showed malignant tumour centred in a bronchial wall and lumen, spreading outwards into the lung. The tumour cells were plump and eosinophilic, arranged in sheets and columns around delicate sinusoidal vessels, and strongly resembled the appearances of the tumour from the first case (fig 1). The retroperitoneal mass was composed of similar tumour and a diagnosis of primary adrenal cortical carcinoma with metastasis to the bronchial wall was made.

There was no evidence of endocrinological disturbance but testosterone and cortisol concentrations were not determined. The patient died 20 months later with osseous metastases; no necropsy was performed.

\section{Discussion}

The tumours in both of these patients presented as apparent bronchial carcinomas. Bronchial metastasis is rare in adrenal carcinoma and in an extensive review ${ }^{1}$ only a single case is cited; this was also a solitary bronchial metastasis. Metastatic adrenal carcinoma within the lung parenchyma is much commoner and often multiple. The incidence of 


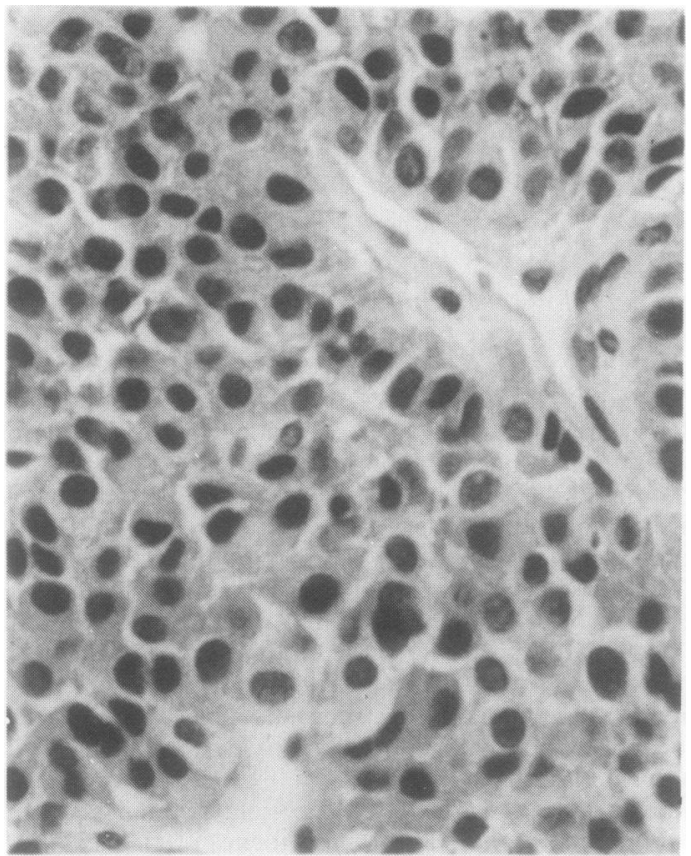

Fig 1 Eosinophilic polygonal cells with nuclear pleomorphism, arranged around delicate sinusoidal blood vessels. (Haematoxylin and eosin, $\times 350$.)

combined pulmonary and osseous metastasis in one series was $24 \%$ at presentation, rising to $79 \%$ at the time of necropsy. ${ }^{2}$ In another report a radiological appearance similar to that in our case 1 led to a course of radiotherapy to a presumed primary bronchial tumour, but subsequent necropsy showed an unsuspected adrenal carcinoma.

Secondary tumours are often symptomless ${ }^{4}$ but an intrabronchial growth is more likely to lead to obstruction or haemoptysis, ${ }^{56}$ mimicking a foreign body or primary carcinoma. The lesions probably arise as a result of haematogenous spread by embolisation to the vessels of the bronchial wall, ${ }^{7}$ and subsequent growth into the air filled bronchial lumen may be easier than spread through solid tissue. ${ }^{8}$ Secondary bronchial tumours are commonly of breast, colonic, renal, or pancreatic origin; rare primary tumours include carcinomas of prostate and bladder, melanoma, choriocarcinoma, and uterine leiomyosarcoma. ${ }^{4} 4$ 10

In view of the necrosis of the tumours, the initial histological diagnosis was difficult in the two cases described here. The pattern of eosinophilic, polygonal, plump cells arranged around delicate, thin walled, sinusoidal vessels was, however, similar in the two cases and an adrenal origin should be suspected when biopsy specimens from single bronchial masses show this appearance.

I should like to thank Drs B Fox and J McIvor for their

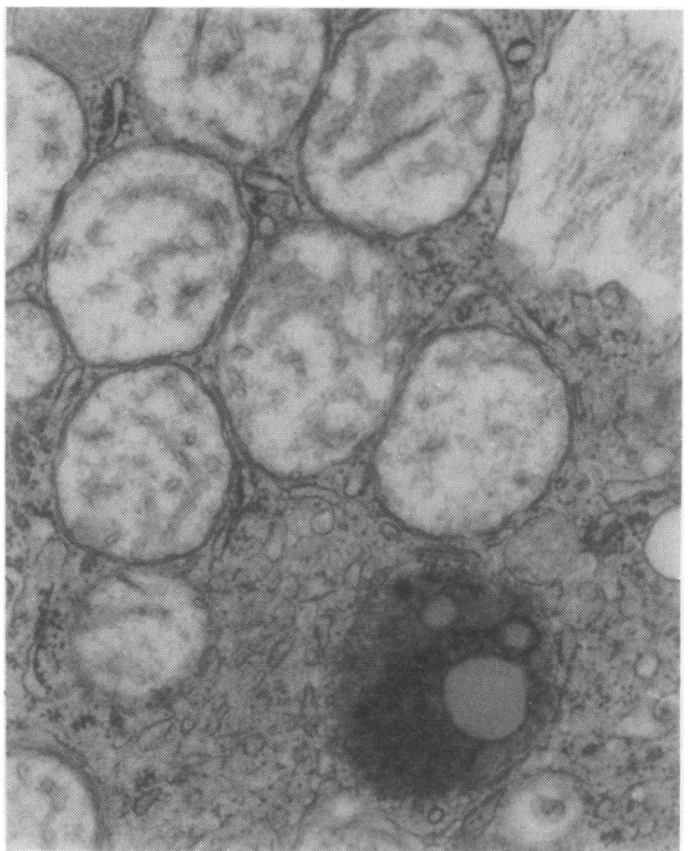

Fig 2 Electron micrograph of tumour cell cytoplasm showing mitochondria with tubular and vesicular cristae; a lipofuscin body with lipid droplets is seen in the lower part of the figure. (Formalin fixed $\times 29700$.)

help, Dr WA Seed and Professor KD Bagshawe for permission to report the first case, Professor G Weber (University of Siena) for the second case, and Miss Emma Barton for secretarial assistance.

\section{References}

'Braman SS, Whitcomb ME. Endobronchial metastasis. Arch Intern Med 1975;135:543-7.

${ }^{2}$ King DR, Lack EE. Adrenal cortical carcinoma, a clinical and pathological study of 49 cases. Cancer $1979 ; 44: 239-44$.

${ }^{3}$ Macfarlane DA. Carcinoma of the adrenal cortex, the natural history, progression and treatment in a study of 55 cases. Ann $R$ Coll Surg Engl 1958;23:155-86.

${ }^{4}$ Marks P, Ferrag MZ, Ashraf H. Rationale for the surgical treat- $\mathcal{N}$ ment of pulmonary metastases. Thorax 1981;36:679-82.

5 Amer E, Guy J, Vase B. Endobronchial metastasis from renal adenocarcinoma simulating a foreign body. Thorax 1981;36:183-4.

- Gerle RG, Fenson B. Metastatic endobronchial hypernephroma. Dis Chest 1963;44:225-33.

7 Anonymous. Many faces of metastases. Lancet 1980;i:748-9.

\& Jariwalla AG, Seaton A, McCormack RGM, Gibbs A, Campbell IA, Davies BH. Intrabronchial metastases from renal carcinoma with recurrent tumour expectoration. Thorax 1981;36:179-82.

'Shepherd MP. Endobronchial metastatic disease. Thorax 1982;37:362-5.

${ }^{10}$ Thomford NR, Woolner LB, Clagett OT. The surgical treatment of metastatic tumours in the lungs. $J$ Thorac Cardiovasc Surg 1965;49:357-63. 